NBER WORKING PAPER SERIES

CENSORED QUANTILE INSTRUMENTAL VARIABLE ESTIMATION WITH STATA

Victor Chernozhukov

Iván Fernández-Val

Sukjin Han

Amanda Kowalski

Working Paper 24232

http://www.nber.org/papers/w24232

NATIONAL BUREAU OF ECONOMIC RESEARCH

1050 Massachusetts Avenue

Cambridge, MA 02138

January 2018

We would like to thank Blaise Melly for helpful comments. The views expressed herein are those of the authors and do not necessarily reflect the views of the National Bureau of Economic Research.

NBER working papers are circulated for discussion and comment purposes. They have not been peer-reviewed or been subject to the review by the NBER Board of Directors that accompanies official NBER publications.

(C) 2018 by Victor Chernozhukov, Iván Fernández-Val, Sukjin Han, and Amanda Kowalski. All rights reserved. Short sections of text, not to exceed two paragraphs, may be quoted without explicit permission provided that full credit, including $\odot$ notice, is given to the source. 
Censored Quantile Instrumental Variable Estimation with Stata

Victor Chernozhukov, Iván Fernández-Val, Sukjin Han, and Amanda Kowalski

NBER Working Paper No. 24232

January 2018

JEL No. C26,C31,C34

\section{ABSTRACT}

Many applications involve a censored dependent variable and an endogenous independent variable. Chernozhukov, Fernandez-Val, and Kowalski (2015) introduced a censored quantile instrumental variable estimator (CQIV) for use in those applications, which has been applied by Kowalski (2016), among others. In this article, we introduce a Stata command, cqiv, that simplifes application of the CQIV estimator in Stata. We summarize the CQIV estimator and algorithm, we describe the use of the cqiv command, and we provide empirical examples.

Victor Chernozhukov

Department of Economics

Massachusetts Institute of Technology

77 Massachusetts Avenue

Cambridge, Mass. 02139

vchern@mit.edu

Iván Fernández-Val

Department of Economics

Boston University

270 Bay State Rd

Boston, MA 02215

ivanf@bu.edu
Sukjin Han

Department of Economics

University of Texas

2225 Speedway, BRB 3.152

Austin, TX 78712

sukjin.han@austin.utexas.edu

Amanda Kowalski

Department of Economics

Yale University

37 Hillhouse Avenue

Box 208264

New Haven, CT 06520

and NBER

amanda.kowalski@yale.edu 


\title{
Censored quantile instrumental variable estimation with Stata
}

\author{
Victor Chernozhukov \\ MIT \\ Cambridge, Massachusetts \\ vchern@mit.edu
}

\author{
Ivan Fernandez-Val \\ Boston University \\ Boston, Massachusetts \\ ivanf@bu.edu \\ Amanda Kowalski \\ Yale University \\ New Haven, Connecticut \\ amanda.kowalski@yale.edu
}

\author{
Sukjin Han \\ UT Austin \\ Austin, Texas \\ sukjin.han@austin.utexas.edu
}

\begin{abstract}
Many applications involve a censored dependent variable and an endogenous independent variable. Chernozhukov et al. (2015) introduced a censored quantile instrumental variable estimator (CQIV) for use in those applications, which has been applied by Kowalski (2016), among others. In this article, we introduce a Stata command, cqiv, that simplifes application of the CQIV estimator in Stata. We summarize the CQIV estimator and algorithm, we describe the use of the cqiv command, and we provide empirical examples.
\end{abstract}

Keywords: st0001, cqiv, quantile regression, censored data, endogeneity, instrumental variable, control function.

\section{Introduction}

Chernozhukov et al. (2015) introduced a censored quantile instrumental variable (CQIV) estimator. In this article, we introduce a Stata command, cqiv, that implements the CQIV estimator in Stata. Our goal is to facilitate the use of the cqiv command in a wide set of applications.

Many applications involve censoring as well as endogeneity. For example, suppose that we are interested in the price elasticity of medical expenditure, as in Kowalski (2016). Medical expenditure is censored from below at zero, and the price of medical care is endogenous to the level of medical expenditure through the structure of the insurance contract. Given an instrument for the price of medical care, the CQIV estimator facilitates estimation of the price elasticity of expenditure on medical care in a way that addressess censoring as well as endogeneity.

The CQIV estimator addresses censoring using the censored quantile regression (CQR) approach of Powell (1986), and it addresses endogeneity using a control function approach. For computation, the CQIV estimator adapts the Chernozhukov and Hong (2002) algorithm for CQR estimation. An important side feature of the cqiv stata command is that it can also be used in quantile regression applications that do not include censoring or endogeneity.

In section 2, we summarize the theoretical background on the CQIV command, following Chernozhukov et al. (2015). In section 3, we introduce the use of the CQIV command. We provide an empirical application with examples that involve estimation of Engel curves, as in Chernozhukov et al. (2015).

\section{Censored quantile IV estimation}

Assumption 1. (Model) We observe $\left\{Y_{i}, D_{i}, W_{i}, Z_{i}, C_{i}\right\}_{i=1}^{n}$, a sample of size $n$ of independent and identically distributed observations from the random vector $(Y, D, W, Z, C)$, which obeys the model 
assumptions

$$
\begin{aligned}
Y & =\max \left(Y^{*}, C\right), \\
Y^{*} & =Q_{Y^{*}}(U \mid D, W, V)=X^{\prime} \beta_{0}(U), \\
D & =Q_{D}(V \mid W, Z),
\end{aligned}
$$

where $X=x(D, W, V)$ with $x(D, W, V)$ being a vector of transformations of $(D, W, V), Q_{Y^{*}}(u$ $D, W, V)$ is the $u$-quantile of $Y^{*}$ conditional on $(D, W, V), Q_{D}(v \mid W, Z)$ is the $v$-quantile of $D$ conditional on $(W, Z)$, and

$$
\begin{aligned}
U & \sim U(0,1) \mid D, W, Z, V, C, \\
V & \sim U(0,1) \mid W, Z, C .
\end{aligned}
$$

Assumption 1 considers a triangular system, where $Y^{*}$ is a continuous latent response variable, $Y$ is an observed response variable obtained by censoring $Y^{*}$ from below at the level determined by the variable $C, D$ is the continuous regressor of interest, $W$ is a vector of covariates, possibly containing $C, V$ is a latent unobserved variable that accounts for the possible endogeneity of $D$, and $Z$ is a vector of "instrumental variables" excluded from the equation for $Y^{*}{ }^{1}$

Under Assumption 1, Chernozhukov et al. (2015) introduce the estimator for the parameter $\beta_{0}(u)$ as

$$
\widehat{\beta}(u)=\arg \min _{\beta \in \mathbb{R}^{\mathrm{dim}(X)}} \frac{1}{n} \sum_{i=1}^{n} 1\left(\widehat{S}_{i}^{\prime} \widehat{\gamma}>\varsigma\right) \rho_{u}\left(Y_{i}-\widehat{X}_{i}^{\prime} \beta\right),
$$

where $\rho_{u}(z)=(u-1(z<0)) z$ is the asymmetric absolute loss function of Koenker and Bassett (1978), $\widehat{X}_{i}=x\left(D_{i}, W_{i}, \widehat{V}_{i}\right), \widehat{S}_{i}=s\left(\widehat{X}_{i}, C_{i}\right), s(X, C)$ is a vector of transformations of $(X, C), \varsigma$ is a positive cut-off, and $\widehat{V}_{i}$ is an estimator of $V_{i}$ which is described below.

The estimator in (4) adapts the algorithm of Chernozhukov and Hong (2002) developed for the censored quantile regression (CQR) estimator to a setting where there is possible endogeneity. As described in Chernozhukov et al. (2015), this algorithm is based on the following implication of the model:

$$
P\left(Y \leq X^{\prime} \beta_{0}(u) \mid X, C, X^{\prime} \beta_{0}(u)>C\right)=P\left(Y^{*} \leq X^{\prime} \beta_{0}(u) \mid X, C, X^{\prime} \beta_{0}(u)>C\right)=u,
$$

provided that $P\left(X^{\prime} \beta_{0}(u)>C\right)>0$. In other words, $X^{\prime} \beta_{0}(u)$ is the conditional $u$-quantile of the observed outcome for the observations for which $X^{\prime} \beta_{0}(u)>C$, i.e., the conditional $u$-quantile of the latent outcome is above the censoring point. These observations change with the quantile index and may include censored observations. Chernozhukov et al. (2015) refer to them as the "quantileuncensored" observations. The multiplier $1\left(\widehat{S}_{i}^{\prime} \widehat{\gamma}>\varsigma\right)$ is a selector that predicts if observation $i$ is quantile-uncensored. For the conditions on this selector, consult Assumptions 4(a) and 5 in Chernozhukov et al. (2015).

cqiv implements the censored quantile instrumental variable (CQIV) estimation which is computed using an iterative procedure where each step takes the form specified in equation (4) with a particular choice of $1\left(\widehat{S}_{i}^{\prime} \widehat{\gamma}>\varsigma\right)$. We briefly describe this procedure here and then provide a practical algorithm in the next section. The procedure first selects the set of quantile-uncensored observations by estimating the conditional probabilities of censoring using a flexible binary choice

\footnotetext{
${ }^{1}$ We consider a single endogenous regressor $D$ in the model and in the cqiv procedure.
} 
model. Since $\left\{X^{\prime} \beta_{0}(u)>C\right\} \equiv\left\{P\left(Y^{*} \leq C \mid X, C\right)<u\right\}$, quantile-uncensored observations have conditional probability of censoring lower than the quantile index $u$. The linear part of the conditional quantile function, $X_{i}^{\prime} \beta_{0}(u)$, is estimated by standard quantile regression using the sample of quantile-uncensored observations. Then, the procedure updates the set of quantile-uncensored observations by selecting those observations with conditional quantile estimates that are above their censoring points, $X_{i}^{\prime} \widehat{\beta}(u)>C_{i}$, and iterate.

cqiv provides different ways of estimating $V$, which can be chosen with option firststage (string). Note that if $Q_{D}(v \mid W, Z)$ is invertible in $v$, the control variable has several equivalent representations:

$$
V=\vartheta_{0}(D, W, Z) \equiv F_{D}(D \mid W, Z) \equiv Q_{D}^{-1}(D \mid W, Z) \equiv \int_{0}^{1} 1\left\{Q_{D}(v \mid W, Z) \leq D\right\} d v,
$$

where $F_{D}(D \mid W, Z)$ is the distribution of $D$ conditional on $(W, Z)$. For any estimator of $F_{D}(D \mid$ $W, Z)$ or $Q_{D}(V \mid W, Z)$, denoted by $\widehat{F}_{D}(D \mid W, Z)$ or $\widehat{Q}_{D}(V \mid W, Z)$, based on any parametric or semi-parametric functional form, the resulting estimator for the control variable is

$$
\widehat{V}=\widehat{\vartheta}(D, W, Z) \equiv \widehat{F}_{D}(D \mid W, Z) \text { or } \widehat{V}=\widehat{\vartheta}(D, W, Z) \equiv \int_{0}^{1} 1\left\{\widehat{Q}_{D}(v \mid W, Z) \leq D\right\} d v
$$

Let $R=r(W, Z)$ with $r(W, Z)$ being a vector of transformations of $(W, Z)$. When string is quantile, a quantile regression model is assumed, where $Q_{D}(v \mid W, Z)=R^{\prime} \pi_{0}(v)$ and

$$
V=\int_{0}^{1} 1\left\{R^{\prime} \pi_{0}(v) \leq D\right\} d v
$$

The estimator of $V$ then takes the form

$$
\widehat{V}=\tau+\int_{\tau}^{1-\tau} 1\left\{R^{\prime} \widehat{\pi}(v) \leq D\right\} d v
$$

where $\widehat{\pi}(v)$ is the Koenker and Bassett (1978) quantile regression estimator which is calculated within cqiv using the built-in qreg command in Stata, and and $\tau$ is a small positive trimming constant that avoids estimation of tail quantiles. The integral in (6) can be approximated numerically using a finite grid of quantiles. ${ }^{2}$ Specifically, the fitted values for pre-specified quantile indices (whose number $n_{q}$ is controlled by option nquant (\#)) are calculated, which then yields

$$
\widehat{V}_{i}=\frac{1}{n_{q}} \sum_{j=1}^{n_{q}} 1\left\{R_{i}^{\prime} \widehat{\pi}\left(v_{j}\right) \leq D_{i}\right\} .
$$

For other related quantile regression models that can alternatively be used, see Chernozhukov et al. (2015).

When string is distribution, $\vartheta_{0}$ is estimated using distribution regression. In this case we consider a semiparametric model for the conditional distribution of $D$ to construct a control variable

$$
V=F_{D}(D \mid W, Z)=\Lambda\left(R^{\prime} \pi_{0}(D)\right)
$$

\footnotetext{
${ }^{2}$ The use of the integral to obtain a generalized inverse is convenient to avoid monotonicity problems in $v \mapsto R^{\prime} \widehat{\pi}(v)$ due to misspecification or sampling error. Chernozhukov et al. (2010) developed asymptotic theory for this estimator.
} 
where $\Lambda$ is a probit or logit link function; this can be chosen using option ldv1 (string) where string is either probit or logit. The estimator takes the form

$$
\widehat{V}=\Lambda\left(R^{\prime} \widehat{\pi}(D)\right),
$$

where $\widehat{\pi}(d)$ is the maximum likelihood estimator of $\pi_{0}(d)$ at each $d$ (see, e.g., Foresi and Peracchi (1995), and Chernozhukov et al. (2013)). ${ }^{3}$ The expression (7) can be approximated by considering a finite grid of evenly-spaced thresholds for the conditional distribution function of $D$, where the number of thresholds $n_{t}$ is controlled by option nthresh(\#). Concretely, for threshold $d_{j}$ with $j=1, \ldots, n_{t}$,

$$
\widehat{V}_{i}=\Lambda\left(R_{i}^{\prime} \widehat{\pi}\left(d_{j}\right)\right), \quad \text { for } i \text { 's s.t. } d_{j-1} \leq D_{i} \leq d_{j} \text { with } d_{0}=-\infty \text { and } d_{n_{t}}=\infty,
$$

where $\widehat{\pi}\left(d_{j}\right)$ is probit or logit estimate with $\tilde{D}_{i}\left(d_{j}\right)=1\left\{D_{i} \leq d_{j}\right\}$ as a dependent variable and $R_{i}$ as regressors.

Lastly, when string is ols, a linear regression model $D=R^{\prime} \pi_{0}+V$ is assumed and $\widehat{V}$ is a transformation of the OLS residual:

$$
\widehat{V}_{i}=\Phi\left(\left(D_{i}-R_{i}^{\prime} \widehat{\pi}\right) / \widehat{\sigma}\right),
$$

where $\Phi$ is the standard normal distribution, $\widehat{\pi}$ is the OLS estimator of $\pi_{0}$, and $\widehat{\sigma}$ is the estimator of the error standard deviation. In estimation of (4) using cqiv, we assume that the control function $\widehat{V}$ enters the equation through $\Phi^{-1}(\widehat{V})$. To motivate this, consider a simple version of the model $(2)-(3)$ :

$$
Y^{*}=\beta_{00}+\beta_{01} D+\beta_{02} W+\Phi^{-1}(\epsilon), \quad \epsilon \sim U(0,1)
$$

where $\Phi^{-1}$ denotes the quantile function of the standard normal distribution, and also assume that $\left(\Phi^{-1}(V), \Phi^{-1}(\epsilon)\right)$ is jointly normal with correlation $\rho_{0}$. From the properties of the multivariate normal distribution, $\Phi^{-1}(\epsilon)=\rho_{0} \Phi^{-1}(V)+\left(1-\rho_{0}^{2}\right)^{1 / 2} \Phi^{-1}(U)$, where $U \sim U(0,1)$. This result yields a specific expression for the conditional quantile function of $Y^{*}$ :

$$
Q_{Y^{*}}(U \mid D, W, V)=X^{\prime} \beta_{0}(U)=\beta_{00}+\beta_{01} D+\beta_{02} W+\rho_{0} \Phi^{-1}(V)+\left(1-\rho_{0}^{2}\right)^{1 / 2} \Phi^{-1}(U),
$$

where $V$ enters the equation through $\Phi^{-1}(V)$.

\subsection{CQIV algorithm}

The algorithm recommended in Chernozhukov et al. (2015) to obtain CQIV estimates is similar to Chernozhukov and Hong (2002), but it additionally has an initial step to estimate the control variable $V$. This step is numbered as 0 to facilitate comparison with the Chernozhukov and Hong (2002) 3-Step CQR algorithm.

For each desired quantile $u$, perform the following steps:

0. Obtain $\widehat{V}_{i}=\widehat{\vartheta}\left(D_{i}, W_{i}, Z_{i}\right)$ from (6), (7) or (8) and construct $\widehat{X}_{i}=x\left(D_{i}, W_{i}, \widehat{V}_{i}\right)$.

1. Select a set of quantile-uncensored observations $J_{0}=\left\{i: \Lambda\left(\widehat{S}_{i}^{\prime} \widehat{\delta}\right)>1-u+k_{0}\right\}$, where $\Lambda$ is a known link function, $\widehat{S}_{i}=s\left(\widehat{X}_{i}, C_{i}\right), s$ is a vector of transformations, $k_{0}$ is a cut-off such that $0<k_{0}<u$, and $\widehat{\delta}=\arg \max _{\delta \in \mathbb{R}^{\operatorname{dim}(S)}} \sum_{i=1}^{n}\left\{1\left(Y_{i}>C_{i}\right) \log \Lambda\left(\widehat{S}_{i}^{\prime} \delta\right)+1\left(Y_{i}=C_{i}\right) \log \left[1-\Lambda\left(\widehat{S}_{i}^{\prime} \delta\right)\right]\right\}$.

\footnotetext{
${ }^{3}$ Chernozhukov et al. (2013) developed asymptotic theory for this estimator.
} 
2. Obtain the 2-step CQIV coefficient estimates: $\widehat{\beta}^{0}(u)=\arg \min _{\beta \in \mathbb{R}^{\operatorname{dim}(X)}} \sum_{i \in J_{0}} \rho_{u}\left(Y_{i}-\widehat{X}_{i}^{\prime} \beta\right)$, and update the set of quantile-uncensored observations, $J_{1}=\left\{i: \widehat{X}_{i}^{\prime} \widehat{\beta}^{0}(u)>C_{i}+\varsigma_{1}\right\}$.

3. Obtain the 3-step CQIV coefficient estimates $\widehat{\beta}^{1}(u)$, solving the same minimization program as in step 2 with $J_{0}$ replaced by $J_{1} \cdot{ }^{4}$

Remark 1 (Step 1). To predict the quantile-uncensored observations, a probit, logit, or any other model that fits the data well can be used. cqiv provides option ldv2 (string) where string can be either probit or logit. Note that the model does not need to be correctly specified; it suffices that it selects a nontrivial subset of observations with $X_{i}^{\prime} \beta_{0}(u)>C_{i}$. To choose the value of $k_{0}$, it is advisable that a constant fraction of observations satisfying $\Lambda\left(\widehat{S}_{i}^{\prime} \widehat{\delta}\right)>1-u$ are excluded from $J_{0}$ for each quantile. To do so, one needs to set $k_{0}$ as the $q_{0}$ th quantile of $\Lambda\left(\widehat{S}_{i}^{\prime} \widehat{\delta}\right)$ conditional on $\Lambda\left(\widehat{S}_{i}^{\prime} \widehat{\delta}\right)>1-u$, where $q_{0}$ is a percentage $(10 \%$ worked well in our simulation with little sensitivity to values between 5 and 15\%). The value for $q_{0}$ can be chosen with option drop1(\#).

Remark 2 (Step 2). To choose the cut-off $\varsigma_{1}$, it is advisable that a constant fraction of observations satisfying $\widehat{X}_{i}^{\prime} \widehat{\beta}^{0}(u)>C_{i}$ are excluded from $J_{1}$ for each quantile. To do so, one needs to set $\varsigma_{1}$ to be the $q_{1}$ th quantile of $\widehat{X}_{i}^{\prime} \widehat{\beta}^{0}(u)-C_{i}$ conditional on $\widehat{X}_{i}^{\prime} \widehat{\beta}^{0}(u)>C_{i}$, where $q_{1}$ is a percentage less than $q_{0}$ (3\% worked well in our simulation with little sensitivity to values between 1 and $\left.5 \%\right)$. The value for $q_{1}$ can be chosen with option drop2(\#). ${ }^{5}$

Remark 3 (Steps 1 and 2). In terms of the notation of (4), the selector of Step 1 can be expressed as $1\left(\widehat{S}_{i}^{\prime} \widehat{\gamma}>\varsigma_{0}\right)$, where $\widehat{S}_{i}^{\prime} \widehat{\gamma}=\widehat{S}_{i}^{\prime} \widehat{\delta}-\Lambda^{-1}(1-u)$ and $\varsigma_{0}=\Lambda^{-1}\left(1-u+k_{0}\right)-\Lambda^{-1}(1-u)$. The selector of Step 2 can also be expressed as $1\left(\widehat{S}_{i}^{\prime} \widehat{\gamma}>\varsigma_{1}\right)$, where $\widehat{S}_{i}=\left(\widehat{X}_{i}^{\prime}, C_{i}\right)^{\prime}$ and $\widehat{\gamma}=\left(\widehat{\beta}^{0}(u)^{\prime},-1\right)^{\prime}$.

\subsection{Weighted Bootstrap Algorithm}

Chernozhukov et al. (2015) recommend obtaining confidence intervals through a weighted bootstrap procedure, though analytical formulas can also be used. If the estimation runs quickly on the desired sample, it is straightforward to rerun the entire CQIV algorithm $B$ times weighting all the steps by the bootstrap weights. To speed up the computation, a procedure is proposed that uses a one-step CQIV estimator in each bootstrap repetition.

For $b=1, \ldots, B$, repeat the following steps:

1. Draw a set of weights $\left(e_{1 b}, \ldots, e_{n b}\right)$ i.i.d from the standard exponential distribution or another distribution that satisfies Assumption 6.

2. Reestimate the control variable in the weighted sample, $\widehat{V}_{i b}^{e}=\widehat{\vartheta}_{b}^{e}\left(D_{i}, W_{i}, Z_{i}\right)$, and construct $\widehat{X}_{i b}^{e}=x\left(D_{i}, W_{i}, \widehat{V}_{i b}^{e}\right)$.

\footnotetext{
${ }^{4}$ As an optional fourth step, one can update the set of quantile-uncensored observations $J_{2}$ replacing $\widehat{\beta}^{0}(u)$ by $\widehat{\beta}^{1}(u)$ in the expression for $J_{1}$ in step 2 , and iterate this and the previous step a bounded number of times. This optional step is not incorporated in cqiv command, as Chernozhukov et al. (2015) find little gain of iterating in terms of bias, root mean square error, and value of Powell objective function in their simulation exercise.

${ }^{5}$ In practice, it is desirable that $J_{0} \subset J_{1}$. If this is not the case, Chernozhukov et al. (2015) recommend altering $q_{0}, q_{1}$, or the specification of the regression models. At each quantile, the percentage of observations from the full sample retained in $J_{0}$, the percentage of observations from the full sample retained in $J_{1}$, and the percentage of observations from $J_{0}$ not retained in $J_{1}$ can be computed as simple robustness diagnostic tests. The estimator $\widehat{\beta}^{0}(u)$ is consistent but will be inefficient relative to the estimator obtained in the subsequent step because it uses a smaller conservative subset of the quantile-uncensored observations if $q_{0}>q_{1}$.
} 
3. Estimate the weighted quantile regression: $\widehat{\beta}_{b}^{e}(u)=\arg \min _{\beta \in \mathbb{R}^{\operatorname{dim}(X)}} \sum_{i \in J_{1 b}} e_{i b} \rho_{u}\left(Y_{i}-\beta^{\prime} \widehat{X}_{i b}^{e}\right)$, where $J_{1 b}=\left\{i: \widehat{\beta}(u)^{\prime} \widehat{X}_{i b}^{e}>C_{i}+\varsigma_{1}\right\}$, and $\widehat{\beta}(u)$ is a consistent estimator of $\beta_{0}(u)$, e.g., the 3 -stage CQIV estimator $\widehat{\beta}^{1}(u)$.

Remark 4 (Step 2). The estimate of the control function $\widehat{\vartheta}_{b}^{e}$ can be obtained by weighted least squares, weighted quantile regression, or weighted distribution regression, depending upon which string is chosen among ols, quantile, or distribution in option firststage(string).

Remark 5 (Step 3). A computationally less expensive alternative is to set $J_{1 b}=J_{1}$ in all the repetitions, where $J_{1}$ is the subset of selected observations in Step 2 of the CQIV algorithm. This alternative is not considered in the cqiv routine, because while it is computationally faster, it sacrifices accuracy.

\section{The cqiv command}

\subsection{Syntax}

The syntax for cqiv is as follows:

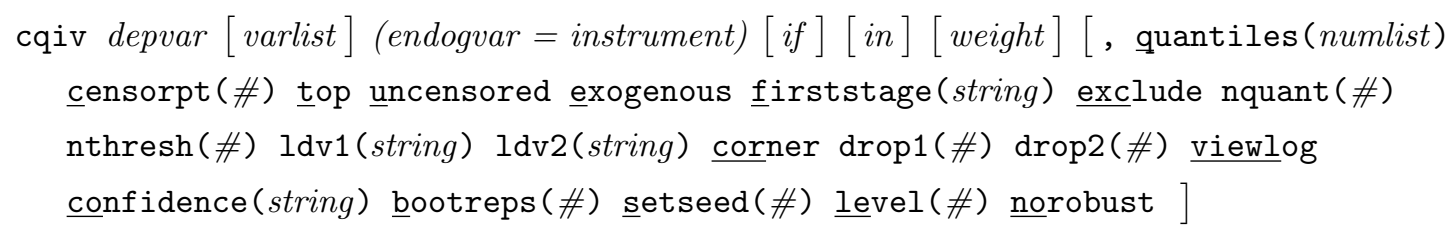

\subsection{Description}

cqiv conducts CQIV estimation. This command can implement both censored and uncensored quantile IV estimation either under exogeneity or endogeneity. The estimators proposed by Chernozhukov et al. (2015) are used if CQIV estimation or QIV without censoring estimation are implemented. The estimator proposed by Chernozhukov and Hong (2002) is used if CQR is estimated without endogeneity. Note that all the variables in the parentheses of the syntax are those involved in the first stage estimation of CQIV and QIV.

\subsection{Option}

\section{Model}

quantiles (numlist) specifies the quantiles at which the model is estimated and should contain percentage numbers between 0 and 100. Note that this is not the list of quantiles for the first stage estimation with quantile specification.

censorpt (\#) specifies the censoring point of the dependent variable, where the default is 0; inappropriately specified censoring point will generate errors in estimation.

top sets right censoring of the dependent variable; otherwise, left censoring is assumed as default.

uncensored selects uncensored quantile IV (QIV) estimation. 
exogenous selects censored quantile regression (CQR) with no endogeneity, which is proposed by Chernozhukov and Hong (2002).

firststage (string) determines the first stage estimation procedure, where string is either quantile for quantile regression (the default), distribution for distribution regression (either probit or logit), or ols for OLS estimation. Note that firststage(distribution) can take a considerable amount of time to execute.

exclude excludes exogenous regressors other than instruments from the first stage estimation.

nquant (\#) determines the number of quantiles used in the first stage estimation when the estimation procedure is quantile; default is 50 , that is, total 50 evenly-spaced quantiles from $1 / 51$ to $50 / 51$ are chosen in the estimation; it is advisable to choose a value between 20 to 100 .

nthresh(\#) determines the number of thresholds used in the first stage estimation when the estimation procedure is distribution; default is 50 , that is, total 50 evenly-spaced thresholds (i.e., the sample quantiles of depvar) are chosen in the estimation; it is advisable to choose a value between 20 and the value of the sample size.

ldv1 (string) determines the LDV model used in the first stage estimation when the estimation procedure is distribution, where string is either probit for probit estimation (the default), or logit for logit estimation.

Idv2 (string) determines the LDV model used in the first step of the second stage estimation, where string is either probit (the default), or logit.

\section{CQIV estimation}

corner calculates the (average) marginal quantile effects for censored dependent variable when the censoring is due to economic reasons such are corner solutions. Under this option, the reported coefficients are the average corner solution marginal effects if the underlying function is linear in the endogenous variable, i.e., the average of $1\left\{Q_{Y^{*}}(U \mid D, W, V)>C\right\} \partial_{D} Q_{Y^{*}}(U \mid D, W, V)=$ $1\left\{x(D, W, V)^{\prime} \beta_{0}(U)>C\right\} \partial_{D} x(D, W, V)^{\prime} \beta_{0}(U)$ over all observations. If the underlying function is nonlinear in the endogenous variable, average marginal effects must be calculated directly from the coefficients without corner option. For details of the related concepts, see Section 2.1 of Chernozhukov et al. (2015). The relevant example can be found in Section 3.5.

drop1(\#) sets the proportion of observations $q_{0}$ with probabilities of censoring above the quantile index that are dropped in the first step of the second stage (See Remark 1 above for details); default is 10 .

drop2 (\#) sets the proportion of observations $q_{1}$ with estimate of the conditional quantile above (below for right censoring) that are dropped in the second step of the second stage (See Remark 2 above for details); default is 3 .

viewlog shows the intermediate estimation results; the default is no log. 


\section{Inference}

confidence(string) specifies the type of confidence intervals. With string being no, which is the default, no confidence intervals are calculated. With string being boot or weightedboot, either nonparametric bootstrap or weighted bootstrap (respectively) confidence intervals are calculated. The weights of the weighted bootstrap are generated from the standard exponential distribution. Note that confidence(boot) and confidence(weightboot) can take a considerable amount of time to execute.

bootreps (\#) sets the number of repetitions of bootstrap or weighted bootstrap if the confidence (boot) or confidence (weightboot) is selected. The default number of repetitions is 100 .

setseed(\#) sets the initial seed number in repetition of bootstrap or weighted bootstrap; the default is 777 .

level(\#) sets confidence level, and default is 95 .

\section{Robust check}

norobust suppresses the robustness diagnostic test results. No diagnostic test results to suppress when uncensored is employed.

\subsection{Saved results}

cqiv saves the following results in e():

Scalars

e (obs) number of observations

e(censorpt) censoring point

e(drop1) $\quad q_{0}$

e(drop2) $\quad q_{1}$

e(bootreps) number of bootstrap or weighted bootstrap repetitions

e(level) significance level of confidence interval

Macros

e (command) name of the command: cqiv

e(regression) name of the implemented regression: either cqiv, qiv, or cqr

e(depvar) name of dependent variable

e(endogvar) name of endogenous regressor

e(instrument) name of instrumental variables

e(firststage) type of first stage estimation

e(confidence) type of confidence intervals

Matrices*

e(results) matrix containing the estimated coefficients, mean, and lower and upper

bounds of confidence intervals

e (quantiles)

row vector containing the quantiles at which CQIV have been estimated

e(robustcheck) matrix containing the results for the robustness diagnostic test results; see

Table B1 of Chernozhukov et al. (2015)

*Note that the entry complete denotes whether all the steps are included in the procedure; 1 when they are, and 0 otherwise. For other entries consult the paper.

\subsection{Examples}

We illustrate how to use the command by using some examples. For the dataset, we use a household expenditure dataset for alcohol consumption drawn from the British Family Expenditure Survey (FES); see Chernozhukov et al. (2015) for detailed description of the data. Using this dataset, we 
are interested in learning how alcohol share of consumption (alcohol) is affected by (logarithm of) expenditure (logexp), controlling for the number of children (nkids). For the endogenous expenditure, we use disposable income, i.e., (logarithm of) wages (logwages), as an excluded instrument. The dataset (alcoholengel.dta) can be downloaded from SSC as follows:

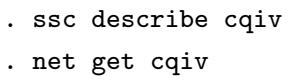

The first line will show the dataset as accessible via the second line of the command. The second line will then download alcoholengel.dta to the current working directory. Given this dataset, we can generate part of the empirical results of Chernozhukov et al. (2015):

- cqiv alcohol logexp2 nkids (logexp = logwages nkids), quantiles(25 50 75) (output omitted)

Using cqiv command, the QIV estimation can be implemented with uncensored option:

- cqiv alcohol logexp2 nkids (logexp = logwages nkids), uncensored (output omitted)

And the CQR estimation with exogenous option:

- cqiv alcohol logexp logexp2 nkids, exogenous

(output omitted)

Here are more possible examples with different specifications and options. Outputs are all omitted.

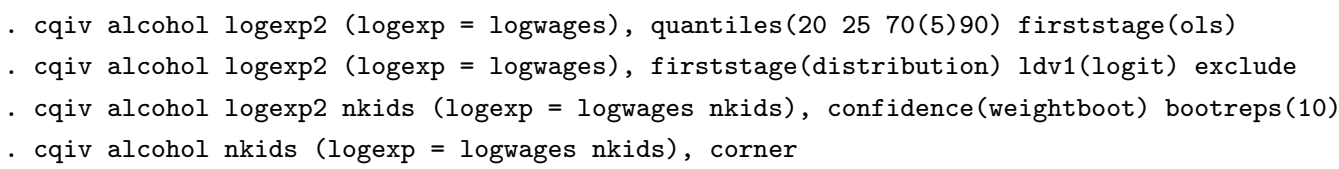

In the second line of the examples, the option exclude is used. In this particular example, logexp2 cannot be included in the first-stage regression when distribution regression is implemented. This is because logexp2 is a monotone transformation of logexp, and thus the distribution estimation yields a perfect fit.

\section{Acknowledgments}

We would like to thank Blaise Melly for helpful comments.

\section{References}

Chernozhukov, V., I. Fernández-Val, and A. Galichon. 2010. Quantile and probability curves without crossing. Econometrica 78(3): 1093-1125.

Chernozhukov, V., I. Fernández-Val, and A. E. Kowalski. 2015. Quantile regression with censoring and endogeneity. Journal of Econometrics 186(1): 201-221.

Chernozhukov, V., I. Fernández-Val, and B. Melly. 2013. Inference on counterfactual distributions. Econometrica 81(6): 2205-2268. 
Chernozhukov, V., and H. Hong. 2002. Three-step censored quantile regression and extramarital affairs. Journal of the American Statistical Association .

Foresi, S., and F. Peracchi. 1995. The conditional distribution of excess returns: An empirical analysis. Journal of the American Statistical Association 90(430): 451-466.

Koenker, R., and G. Bassett, Jr. 1978. Regression quantiles. Econometrica: journal of the Econometric Society 33-50.

Kowalski, A. 2016. Censored Quantile Instrumental Variable Estimates of the Price Elasticity of Expenditure on Medical Care. Journal of Business \& Economic Statistics 34(1): 107-117. URL https://doi.org/10.1080/07350015.2015.1004072.

Powell, J. 1986. Censored regression quantiles. Journal of Econometrics 32(1): 143-155. Cited By 341. URL https://www.scopus.com/inward/record.uri?eid= 2 -s2.0-38249039685\&doi=10.1016\%2f 0304-4076\%2886\% $2990016-3 \&$ partner ID $=40 \& m d 5=$ 65bc49a1ededfc7bebae3335dc029e74. 